\title{
Welcome to Medical Technologies Journal
}

\section{Type of Article: Editorial}

\author{
Abdeldjalil Khelassi \\ Informatics department, sciences faculty, Abou Beker Belkaid University of Tlemcen, \\ Algeria \\ khelassi.a@gmail.com
}

\begin{abstract}
Welcome to Medical Technologies Journal MTJ. MTJ is an international peerreviewed journal and an interdisciplinary one focusing on relevant innovations on medical technologies and findings relative to medicine. This editorial presents the global initiative of the journal in a nutshell. It is also dedicated to welcoming contributions to the journal and for introducing the first issue.
\end{abstract}

Keywords: International publishing, Medical technologies, Open Access, Medical Research, Health Science

\section{Introduction}

I'm very glad to start the journal with this editorial. I'm also delighted to welcome the members of the scientific community to contribute intensively to the journal. With this initiative and your help we aim to serve the humanity by a reputable journal responding to the scientific standards. We hope also to contribute significantly to the scientific and research progress.

Medical Technologies Journal MTJ is an international journal on medical technologies and health sciences. The journal vise a high quality of scholarly publication by applying strong selection rules based on relevancy, originality and attractively of research works. Also the publishing ethics, human and animal rights must be respected.

The journal employs an exceptional editorial team for ensuring the fast replay for all publications. Different types of articles can be scheduled for publication in the future issues like research articles, Original articles, review articles, technical notes, as a primary category. As a secondary articles: editorials, opinions, perspectives, letter to the editor, biography, conference abstract and interview.

The first issue contains selected abstracts from the international conference on health sciences and medical technologies elaborated between 27 and 29 September 2016 at the Abou Beker Belkaid University of Tlemcen, Algeria.

\section{Topics and scopes}

MTJ is an interdisciplinary journal, in which several domains and specialties could contribute by their research works, including but not limited to medicine, information and communication technology, physics, chemistry, mechanical and electrical engineering, biology, sociology and others. 
Although it is very difficult to separate between the specialties of the journal, three sections are defined:

\section{a. Medical technologies}

Including but not limited: Information System, Knowledge engineering, Case-Based Reasoning, Multi-Agent Systems, Web applications, Social Networks, Data Sciences, Distributed Systems, Cloud computing, Biomedical Engineering, Medical physics, Medical Bio-Chemistry, Medical Mechanical Engineering, Image and signal processing, ComputerAided Diagnosis, Bioinformatics, Drug design, Data mining and knowledge discovery, Mobile health, Telemedicine, Health 2.0, Medical education, E-learning for medicine, Assistive Technologies in Medicine, Social insurance medicine.. Etc.

\section{b. Health sciences}

Surgery, Internal Medicine, Midwifery, Nursing, Microbiology and Infectious Diseases, Psychiatry, Pediatrics, Cardiology, Neurology, Nephrology and Urology, Orthopedics, Neurosurgery, Dermatology, Rheumatology, Hematology and Transfusion Science, Anatomy, Histopathology, Oncology, Community Health, Occupational Health, Environmental Health, Social Health, Epidemiology, Public health, Patient Security, ..Etc.

\section{c. Conferences proceedings and abstracts}

All peer-reviewed conferences proceedings and abstracts on the above cited topics.

\section{Acknowledgments}

We would warmly like to thank all contributors, authors, reviewers, and editors, on this issue and we hope more contributions in the future issues.

\section{Declaration of conflicts}

\section{No declaration}

\section{Authors' biography}

Dr Abdeldjalil Khelassi is an associate professor of Informatics at Abou Beker Belkaid University of Tlemcen. The chair of International Conference on Health Sciences and Medical Technologies 2016. Head of Knowledge and Information Engineering Research Team.

\section{References}

No reference 